\title{
EFFECTIVENESS OF TRANSPOSED INVERSE SETS IN FABER REGIONS
}

\author{
J.A. ADEPOJU \\ Department of Mathematics \\ Faculty of Science \\ University of Lagos \\ Lagos, Nigeria \\ M. NASSIF \\ 7 Denchaway Street \\ Sidi Gaber, Alexandria, Egypt \\ (Received Frbruary 11, 1982 and in revised form August 15, 1982)
}

\begin{abstract}
The effectiveness properties, in Faber regions, of the transposed inverse of a given basic set of polynominals, are investigated in the present paper. A certain inevitable normalizing substitution, is first formulated, to be undergone by the given set to ensure the existence of the transposed inverse in the Faber region. The first main result of the present work (Theorem 2.1), on the one hand, provides a lower bound of the class of functions for which the normalized transposed inverse set is effective in the Faber region. On the other hand, the second main result (Theorem 5.2) asserts the fact that the normalized transposed inverse set of a simple set of polynomials, which is effective in a Faber region, should not necessarily be effective there.
\end{abstract}

KEY WORDS AND PHRASES. Basic Sets of Polynomials, Transpose and Transposed Inverse Sets, Effectiveness, Faber Regions.

1980 MATHEMATICS SUBJECT CLASSIFICATION CODE. $41 \mathrm{~A} 10$.

1. INTRODUCTION.

The transposed inverse set of a given basic set of polynomials is the set whose matrix of coefficients is the transposed inverse of that of the given set. This set was introduced by Newns [1; p. 455] who has also studied the relation between the effectiveness in disks of centre origin of the $g$ iven set and that of the transposed in- 
verse set [1; pp. 456, 457]. Moreover, the effect iveness properties in Faber reg ions, of the transpose of simple sets of polynomials effective in the same regions, have been investigated by Nassif [2]. We propose therefore, as a complement of the investigation of Nassif, to consider in the present paper (which is extracted from the Ph:D Thesis of Adepoju [3; Chapter II]), the effectiveness properties, in Faber regions, of the transposed inverse set of a given basic set of polynomials. In particular, we shall study here the extent of generalization, to Faber regions, of the result of Newns referred to above. It should be mentioned here that the reader is supposed to be acquainted with the theory of basic sets of polynomials, as given by Whittaker [4], as well as the properties of Faber regions and Faber polynomials as given by Newns [5; pp. 746 - 751] and the essentials of the theory of effectiveness in Faber regions, of basic sets, as recorded by Newns [1; pp. 452, 453], [6; p. 190]. For sake of further application in our subsequent work, we shall assemble, in what follows, certain results, pertaining to Faber regions. (Detailed proofs of these results can be found in Adepoju's work [3; pp. $50-54$, and $58-60])$.

Suppose, in fact, that the Faber curve $C$ is the map, in the $z$ plane of the circle $|t|=\gamma$, by the Faber transformation

$$
z=k(t) \equiv t+\sum_{m=0}^{\infty} a_{n} t^{-n},
$$

which is supposed to be conformal for $|t|>T$, for some $T>\gamma$. Suppose also that

$$
t=\psi(z)
$$

is the inverse transformation, which is conformal for $|z|>T^{\prime}$, say.

The following notations are adopted throughout the present work.

$$
\begin{gathered}
G(r)=\sup _{\theta}\left|\phi\left(\mathrm{re}^{i \theta}\right)\right| ; \quad(\mathrm{r}>\mathrm{T}), \\
\mathrm{E}(\mathrm{r})=\sup _{\theta}\left|\psi\left(\mathrm{re}^{\mathrm{i} \theta}\right)\right| ; \mathrm{e}(\mathrm{r})=\inf _{\theta}\left|\psi\left(\mathrm{re}^{i \theta}\right)\right| ;\left(\mathrm{r}>\mathrm{T}^{\prime}\right), \\
\sigma=\inf \{\mathrm{G}(\mathrm{r}) ; \mathrm{r}>\mathrm{T}\} ; \mathrm{g}^{-}=\inf \left\{\mathrm{E}(\mathrm{r}) ; \mathrm{r}>\mathrm{T}^{\prime}\right\} .
\end{gathered}
$$

With these notations, the results to be assembled here are as follows

$$
\begin{gathered}
T^{\prime} \leq \rho, T \leq \rho^{\prime} \\
\text { e }\{G(r)\}=r ;(r>T) ; G\{e(r)\}=r ;(r \geq g),
\end{gathered}
$$

and if the Faber curve $\mathrm{C}_{r}$ is the map, in the $z$-plane, of the circle $|t|=r$; 
$\mathrm{T}<\mathbf{r}<\infty$, then, in the usual notation for domains, (c. f. Newns [1; p. 436)],

$$
D(r) \subset D\left\{C_{E(r)}\right\} ;\left(r>T^{\prime}\right)
$$

Also, the following monotony properties will be of constant use in our subsequent work without being explicitly stated. $G(r), E(r)$ and $e(r)$ are continuous strictly increasing functions of $r$ for $r>T, r>T^{\prime}$ and $r \geq \rho$ respectively.

Moreover, if $\left\{\mathrm{f}_{\mathrm{k}}(\mathrm{z})\right\}$ and $\left\{\overline{\mathrm{f}}_{\mathrm{k}}(\mathrm{z})\right\}$ are the $\phi$ - and $\psi$ _ bases respectively, and if we write

$$
M\left(f_{k}, r\right)=\sup _{\theta}\left|f_{k}\left(r e^{i \theta}\right)\right| ; M\left(\bar{f}_{k}, r\right)=\sup _{\theta}\left|\bar{f}_{k}\left(r e^{i \theta}\right)\right|
$$

and put

$$
\left.\mu(r)=\lim _{k \rightarrow \infty} \sup _{k \rightarrow \infty}\left\{M\left(f_{k}, r\right)\right\}^{1 / k} ; \bar{\mu}(r)=\lim _{k \rightarrow \infty} \sup _{k}\left\{\bar{f}_{k}, r\right)\right\}^{1 / k},
$$

then, by appeal to the familiar formulas

$$
\begin{gathered}
\mathrm{f}_{\mathrm{k}}(\mathrm{z})=\{\psi(\mathrm{z})\}^{\mathrm{k}}+\left(\frac{1}{2 i \pi}\right) \int \frac{\{\psi(\zeta)\}^{\mathrm{k}} \mathrm{d} \zeta}{\zeta-\mathrm{z}} ;\left(\mathrm{k} \geq 1 ; \mathrm{z} \in \mathrm{C}_{\mathrm{r}} ; \mathrm{T}^{-}<\mathrm{T}_{1}<\mathrm{r}\right) \\
\overline{\mathrm{f}}_{\mathrm{k}}(\mathrm{z})=\{\phi(\mathrm{z})\}^{\mathrm{k}}+\left(\frac{1}{2 i \pi}\right) \int_{|\mathrm{t}|=\mathrm{T}_{2}} \frac{\{\phi(\mathrm{t})\}^{\mathrm{k}} \mathrm{dt}}{\mathrm{t}-\mathrm{z}} ;\left(\mathrm{k} \geq 1 ;|\mathrm{z}|>\mathrm{T}_{2}>\mathrm{T}\right),
\end{gathered}
$$

we obtain

$$
\mu(r)=E(r) ;\left(r>T^{\prime}\right) ; \bar{\mu}(r)=G(r) ;(r>T) .
$$

2. NORMALIZING SUBSTITUTION.

In our notation, a cap $(\wedge)$ over a set indicates that the set is the transposed inverse of the corresponding set. Our main concern in the present work is to establish a relationship between the effectiveness properties, in Faber regions, of a given set $\left\{\mathrm{p}_{k}(z)\right\}$ and those of the transposed inverse set $\left\{\hat{\mathrm{p}}_{k}(z)\right\}$. As in the case of transposed sets (c.f. [2; p. 212]), to ensure the existence an non-violation of effectiveness properties of the transposed inverse set in Faber regions, the given set has to be normalized in a certain manner. The essentiality of this normalizing process will be made obvious by consideration of the $\phi_{-}$base $\left\{f_{k}(z)\right\}$, which is a simple set effective in all $\mathrm{D}\left(\mathrm{C}_{\mathrm{r}}\right) ; \mathrm{r}>\mathrm{T}$. The transposed inverse of this set is

$$
\hat{\mathrm{f}}_{\mathrm{k}}(\mathrm{z})=-\frac{\psi^{\prime}(1 / \mathrm{z})}{\mathrm{z}\{\psi(1 / \mathrm{z})\}^{k+1}} ;(\mathrm{k} \geq 0),
$$


which may not exist in $D(C)$ for certain - urms of $C$, (as for example when $C$ is a Cassini ova1).

In fact, we write

$$
\alpha=G(\gamma) ; \beta=\alpha\left[\max \left\{\alpha, G\left(g^{\prime}\right)\right\}\right\rceil,
$$

and the normalized set $\left\{\mathrm{P}_{k}(z)\right\}$, is derived from the given set $\left\{\mathrm{P}_{k}(z)\right\}$, through the substitution

$$
\mathrm{P}_{\mathrm{k}}(\mathrm{z})=\mathrm{P}_{\mathrm{k}}(\xi \mathrm{z}) ;(\mathrm{k} \geq 0),
$$

where $\zeta$ is any complex number satisfying

$$
\beta \leq|\xi|<\infty \text {. }
$$

Thus the tranposed inverse set $\left\{\hat{P}_{k}(z)\right\}$ of the set $\left\{P_{k}(z)\right\}$ will be given by

$$
\hat{\mathrm{P}}_{\mathrm{k}}(\mathrm{z})=\hat{\mathrm{p}}_{\mathrm{k}}(\mathrm{z} / \xi) ;(\mathrm{k} \geq 0) \text {. }
$$

It should be observed, beforehand, that the normalizing substitution (2.3) wil1 lead to favourable result when $C$ is a circle of centre origin. In fact, if the given set $\left\{\mathrm{P}_{k}(z)\right\}$ is effective in $D(R) ; R>0$, it can easily be shown that the transposed inverse set $\left\{\hat{P}_{k}(z)\right\}$ of (2.5) will be effective in $D_{+}(|\xi| / R) ;|\xi| \geq R^{2}$.

Suppose now that $\mathrm{C}$ is not a circle of centre origin, as we shall always assume in the subsequent work. To formulate a positive result concerning this case we fix the number $\eta$ by

$$
E(n)=e(|\xi| / \alpha)
$$

It easily follows from (1.2), (1.4), (2.2) and (2.4), that

$$
E(\eta) \geq \gamma ; \eta=T^{\prime}
$$

Hence, we deduce from (1.2) and (1.3), that, for all numbers $\eta^{-}$for which $T^{-}<\eta^{-} \leq \eta_{\text {, }}$ the curve $C_{E\left(n^{\prime}\right)}$ is well defined.

With the above notation, and with the usual notation for functions classes (c. f. Newns [1; p. 436]), we can formulate the first main result of the present work in the following theorem:

THEOREM 2.1. Suppose that there is a number $\eta^{-}$with $T^{-}<\eta^{-} \leq \eta$, such that the given basic set $\left\{\mathrm{P}_{\mathrm{k}}(z)\right\}$ is effective in $D_{+}\left\{C_{E\left(n^{\prime}\right)}\right\}$ for the class of functions $\overline{\mathrm{H}}\left\{\mathrm{C}_{\mathrm{E}(n)}\right\}$. Then the transposed inverse set $\left\{\hat{\mathrm{P}}_{\mathrm{k}}(\mathrm{z})\right\}$ of the nurmalized set $\left\{\mathrm{P}_{\mathrm{k}}(\mathrm{z})\right\}$ of (2.3), will be effective in $D(C)$ for the class of functions $\bar{H}\left(C \sigma^{\prime}\right)$, where

$$
\sigma^{\circ}=\mathrm{E}\left(|\xi| / \eta^{\prime}\right) \text {. }
$$


We first observe from $(1.4),(2.6)$ and $(2.8)$ that $\sigma^{-}>\gamma$, so that the result of the above theorem is legitimate. Moreover, suppose that the given set $\left\{p_{k}(z)\right\}$ is simple and effective in $D_{+}(C)$, then we can derive from the above theorem the following corollary, which is the analogue of the main result concerning the transposed set in Faber region (c. f. [2; Theorem 1]).

COROLLARY 2.2. If $\left\{\mathrm{p}_{\mathrm{k}}(\mathrm{z})\right\}$ is a simple set of polynomials which is effective in $D_{+}(C)$ then the transposed inverse set $\left\{\hat{P}_{k}(Z)\right\}$ will be effective in $D(C)$ for the class of functions $\overline{\mathrm{H}}\left(\mathrm{C}_{\sigma}\right)$, where

$$
\sigma=\mathbf{E}(|\xi| / \eta)
$$

Moreover, we should note that the bound (2.4) for the normalizing factor $\xi$ is justified in the sense that, for certain forms of the Faber curves (C), there exists a simple set $\left\{p_{k}(z)\right\}$ of polynomials which is effective in $D_{+}(C)$, and yet the transposed inverse set $\left\{\hat{P}_{k}(z)\right\}$ of the set $\left\{P_{k}(z)\right\}$ of (2.3), corresponding to values of $\xi$ for which $|\xi|<\beta$, does not exist in $D(C)$. Thisphenomenon is illustrated by example 3.1 below.

It can be further observed that if, for a given basic set $\left\{p_{k}(z)\right\}$, no such numbers $\eta^{\prime}$ exists to accord with the supposition of theorem 2.1, the normalized transposed inverse set $\left\{\hat{P}_{k}(z)\right\}$ may no longer be basic in $D(C)$ for certain forms of the Faber curves (C). This observation will be displayed in example 3.2 below.

Now, according to $(1.4),(2.6)$ and (2.9), we may finally see from corollayr 2.2, that, contrary to the case of disks with centre origin, the transposed inverse set $\left\{\hat{P}_{k}(z)\right\}$ oforresponding to the simple set $\left\{p_{k}(z)\right\}$ of polynomials, which is effective in $D_{+}(C)$, may not be effective in $D(C)$. This vact is ascertained by the second main result of the present work formulated in theorem 5.2 below.

\section{EXAMPLES.}

Basic sets of polynomials are constructed in the present section such that the observations given in the last section are fulfilled.

EXAMPLE 3.1. Let $c$ be a Faber curve for which the number $f^{\prime}$ of $(1.2)$ is 1 ess than $\gamma$ (as for example the Cassini oval $\left|z^{2}-1\right|=c^{2}$, for which $c>\sqrt{2}$ ). Therefore, according to (2.2) we shall have $\beta=\alpha^{2} \quad$ Then we shall take 


$$
\xi=\alpha^{2}(1-\delta),
$$

where $\delta$ is some positive number less than 1. Also, from the definition (2.2) of the number $\alpha$ and in view of (1.1), we can choose a real number $\theta$ for which

$$
\left|\phi\left(\gamma \mathrm{e}^{i \theta}\right)\right|=\alpha \text {. }
$$

We shall show that the transposed inverse set $\left\{\hat{P}_{k}(z)\right\}$ of the set $\left\{P_{k}(z)\right\}$, corresponding to the following simple set $\left\{p_{k}(z)\right\}$, with the number $\xi$ of (3.1) , does not exist in $D(C)$.

$$
p_{0}(z)=f_{0}(z) ; p_{k}(z)=-\gamma^{k} e^{1 k \theta}+f k(z) ;(k \geq 1) \text {. }
$$

It can be verified that this set is effective in $D_{+}(C)$, and that, for the transposed inverse set, we shall formally have

$$
\hat{\mathrm{P}}_{\mathrm{o}}(z)=\frac{\xi \psi^{\prime}(\xi / z)}{z \psi(\xi / z)-\gamma \mathrm{e}^{i \theta}}=\sum_{k=0}^{\infty} \bar{f}_{k}\left(\gamma \mathrm{e}^{i \theta}\right)(z / \xi)^{\mathrm{k}} .
$$

Appealing to the formula (1.7) it can be deduced, from (3.1) and (3.2), that

$$
\underset{k \rightarrow \infty}{\lim \sup _{k \rightarrow \infty}}\left|\vec{f}_{k}\left(\gamma e^{i \theta}\right)\left(z^{-} / \xi\right)^{k}\right|^{1 / k}=\frac{1-\delta / 2}{1-\delta}>1,
$$

where the point $z^{\prime} \in D(C)$ is chosen such that $\left|z^{-}\right|=\alpha(1-\delta / 2)$.

Therefore, the expansion (3.3) of $\hat{\mathrm{P}}_{0}(z)$ is impossible at $z^{\prime}$, as required.

EXAMPLE 3.2. Let $\mathrm{C}$ be a Faber curve of the form considered in example 3.1 and set the finite numbers $x$ and $\rho$ such that

$$
\mathbf{x} \geq \beta
$$

and that

$$
\rho=(1+\delta) \quad e(x / \alpha)
$$

where $\delta$ is, again, any positive number less than 1 . Also, from the continuity and monotony of the function $e(r) ; r \geq \rho$, we can determine the number $r_{1}$ such that

$$
r_{1}>x / \alpha ; e\left(r_{1}\right)<(1+\delta / 2) e(x / \alpha)
$$

With this value of $r_{1}$ we can choose the point $z_{0} \in D(C)$ in such a way that

$$
\left|z_{0}\right|=x / r_{1}<\alpha
$$

Having fixed $z_{0}$, the numver $\xi$ can be defined as 


$$
|\xi|=\mathbf{x} \geq \beta
$$

and

$$
\left|\psi\left(\xi / z_{0}\right)\right|=e\left(r_{1}\right) .
$$

Consider now the set $\left\{p_{k}(z)\right\}$ of polynomials, given as follows.

$$
p_{0}(z)=f_{o}(z) ; p_{k}(z)=-f^{k} f_{o}(z)+f_{k}(z) ;(k \geq 1) \text {. }
$$

It can be verified, from (2.6), (3.5) and (3.7) that, for the set $\left\{p_{k}(z)\right\}$ of (3.9), there is no number $\eta^{\prime}$, with $T^{\prime}<n^{\prime} \leq n$, such that the set is effective in $D_{+}\left\{C_{E\left(n^{\prime}\right)}\right\}$ for the class of functions $\bar{H}\left\{C_{E(n)}\right\}$. We shall show that the transposed inverse set $\left\{\hat{P}_{k}(z)\right\}$ is not vasic in $D(C)$.

In fact, it can be easily proved, from (2.1), (2.5) and (3.9), that

$$
\hat{\mathrm{P}}_{\mathrm{k}}(z)=\frac{\xi \psi^{\prime}(\xi / z)}{z \psi(\xi / z)^{\mathrm{k}+1}} ;(\mathrm{k} \geq 1) \text {. }
$$

Moreøver, the definition (3.9) of the set $\left\{p_{k}(z)\right\}$ implies the representation

$$
1=\hat{\mathrm{p}}_{0}(z)+\sum_{k=1}^{\infty}\left\{\mathrm{fk}(0)-\rho^{\mathrm{k}}\right\} \hat{\mathrm{P}}_{\mathrm{k}}(z)
$$

Now, we can apply the formula (1.6) to deduce, in view of (1.5), that

$$
\left|f_{k}(0)\right| \leq \sup _{z \in C_{T_{1}}}\left|f_{k}(z)\right|<M_{1}^{k} ;(k \geq 1),
$$

where $M$ is a positive finite number independent of $k$, and $T_{1}$ is a number chosen so that $\mathrm{g}^{\prime}<\mathrm{T}_{1}<\gamma$ and this is allowable for the foregoing form of the curve $C$. Also, from (3.8) and (3.10) it follows that

$$
\left|\mathrm{P}_{k}\left(z_{0}\right)\right|=\frac{|\xi| L}{\left|z_{o}\right|\left\{e\left(r_{1}\right)\right\}^{k+1}} ;(k \geq 1) .
$$

where $\mathrm{L}=\left|\psi^{-}\left(\xi / \mathrm{z}_{0}\right)\right|>0$. A combination of (2.7), (3.4), (3.5), (3.6), (3.12) and (3.13) will lead to the following inequality

$$
\begin{gathered}
\left|f_{k}(0)-\rho^{k}\right|\left|P_{k}\left(z_{o}\right)\right| \geq \frac{|\xi| L}{z_{o} \mid e\left(r_{1}\right)}\left(\frac{1+\delta}{1+\delta / 2}\right)^{k}\left\{1-M(1+\delta)^{-k}-\left(T_{1} / \gamma\right)^{k}\right\} \\
>\frac{|\xi| L}{2\left|z_{o}\right| e\left(r_{1}\right)}\left(\frac{1+\delta}{1+\delta / 2}\right)^{k}
\end{gathered}
$$


for all sufficiently large values of $k$. Therefore, the representation (3.11) is impossible at $z_{0} \in D(C)$ and the set $\left\{\hat{P}_{k}(z)\right\}$ is not basic in $D(C)$; as required.

4. PROOF OF THEOREM 2.1. The proof is similar to that of theorem 1 of [2], even more direct. In fact, let $\left\{q_{k}(z)\right\}$ be the set associatiated with the given set $\left\{\mathrm{P}_{\mathbf{k}}(\mathrm{z})\right\}$, which is now given as the product set

$$
\left\{p_{k}(z)\right\}=\left\{q_{k}(z)\right\} \quad\left\{f_{k}(z)\right\}
$$

Denote by $\kappa$ and $\kappa^{(1)}$ for the Cannon functions of the sets $\left\{p_{k}(z)\right\}$ and $\left\{q_{k}(z)\right\}$ respectively. We can now apply the conditions imposed on the set $\left\{p_{k}(z)\right\}$ in theorem 2.1, the properties of the associated set $\left\{q_{k}(z)\right\}$ and the formula (1.8), to obtain

$$
K^{(1)}\left\{\mu\left(n^{\prime}\right)+\right\} \leq E(n) \text {. }
$$

Thus, a combination of the relations $(1.4),(1.8),(2.2),(2.4),(2.6),(2.7)$ and (4.2) will lead to the inequality

$$
\bar{\mu}\left[K^{(1)}\left\{\mu\left(n^{\prime}\right)+\right\}\right] \leq|\xi| / \alpha \text {. }
$$

Therefore, applying an alternative form of Theorem 2 of Newns [6] to the set $\left\{p_{k}(z)\right\}$, given as the product (4.1), then (4.3) yields

$$
\kappa\left(n^{-}+\right) \leq|\xi| / \alpha \text {, }
$$

which expresses the fact that the set $\left\{p_{k}(z)\right\}$ is effective in $D_{+}\left(n^{\prime}\right)$ for the class of functions $\overline{\mathrm{H}}(|\xi| / \alpha)$. Therefore, a modified form of Theorem 16.3 of Newns [1] implies that the transposed inverse set $\left\{\hat{p}_{k}(z)\right\}$ will be effective in $D(\alpha /|\xi|)$ for the class of functions $\bar{H}\left(1 / \eta^{\prime}\right)$. It follows that the set $\left\{\hat{P}_{k}(z)\right\}$, which is in fact, the set $\left\{\hat{P}_{k}(z / \xi)\right\}$, will be effective in $D(\alpha)$ for the class of functions $\bar{H}\left(|\xi| / n^{\prime}\right)$.

Now, in view of $(1.1),(1.2),(1.3),(1.5),(2.6)$ and $(2.8)$ and from the definition of the number $\alpha$, we shall have

$$
\overline{\mathrm{D}}\left(|\xi| / n^{\prime}\right) \subset \overline{\mathrm{D}}\left(\mathrm{C}_{\sigma^{\prime}}\right) \text { and } \mathrm{D}(\mathrm{C}) \subset \mathrm{D}(\alpha) \text {. }
$$

We thus conclude that the set $\left\{\hat{P}_{k}(z)\right\}$ is effective in $D(C)$ for the class of functions $\overline{\mathrm{H}}\left(\mathrm{C}_{\sigma^{-}}\right)$and theorem 2.1 is therefore established. 


\section{NONEFFECTIVENESS OF TRANSPOSED INVERSE SETS.}

As supplement to the positive result involved in theorem 2.1 above, we shall establish in the present section a negative result which is in contrast with the favourable result stated in $\S 2$ concerning the case of disks with centre origin (p. 5, 11. 1-5). Actually, we sha11 prove, in the second main result of the present work formulated in theorem 5.2 below, that, for all forms of the Faber curve C (which is not a circle of centre origin), there are values of the normalizing factor $\xi$, with $|\xi| \geq \beta$, such that the normalized transposed inverse set $\left\{\hat{\mathrm{t}}_{\mathrm{k}}(\mathrm{z} / \xi)\right\}$, derived from the $\phi_{-} b a s e\left\{f_{k}(z)\right\}$, is not effective in $D(C)$.

First of all, from the definition (2.2) of the number $\alpha$, there is a point $\zeta \epsilon \mathrm{C}$ for which

$$
|\zeta|=\alpha
$$

Therefore, according to the equation (2.6), we can choose the number $\xi$, with $|\xi| \geq \beta$, and a real number $\omega$ such that

$$
|\psi(\xi / \zeta)|=\mathrm{e}|\xi| / \alpha=E(n)=\left|\psi n \mathrm{e}^{-i \omega}\right|=\tau,
$$

say. With this determination of the number $\xi$, the following theorem has first to be proved for the transposed inverse set $\left\{\hat{\mathrm{P}}_{\mathrm{k}}(\mathrm{z})\right\}$, given by

$$
\hat{\mathrm{P}}_{\mathrm{k}}(\mathrm{z})=\hat{\mathrm{f}}_{\mathrm{k}}(\mathrm{z} / \xi) ;(\mathrm{k} \geq 0) \text {. }
$$

THEOREM 5.1. For all forms of the Faber curve $C$, the Cannon function $\hat{K}(C)$ of the set $\left\{\hat{P}_{k}(z)\right\}$, for $\bar{D}(C)$, is bounded. In fact, the following inequality is established,

$$
\hat{\kappa}(C) \leq \sigma,
$$

where $\sigma$ is given by (2.9).

It should be first mentioned that the inequality (5.4) cannot be deduced from corollary 2.2 above since the set $\left\{\hat{P}_{k}(z)\right\}$ is not simple.

PROOF. We first observe, from (2.1) and (5.3), that

$$
\hat{\mathrm{P}}_{\mathbf{k}}(\mathrm{z})=\frac{\xi \psi^{\prime}(\xi / z)}{z\{\psi(\xi / z)\}^{\prime}+1} ;(k \geq 0) \text {. }
$$

Therefore, adopting the notation applied before, namely, 


$$
M(f ; r)=\sup _{\theta}\left|f\left(r e^{i \theta}\right)\right|,
$$

for any function $f(z)$ regular on $|z|=r$, we obtain from (5.2) and (5.5),

$$
M\left(\hat{P}_{k} ; \alpha\right) \leq \frac{\left(|\xi| L^{\prime}\right)}{\left(\alpha \tau^{k+1}\right)} ;(k \geq 0),
$$

where $L^{\prime}=\sup \left\{\left|\psi^{\prime}(\xi / z)\right|:|z|=\alpha\right\}<\infty$. Also, it follows from (5.3) and the defirition of transposed inverse sets that

$$
f_{n}(z)=\sum_{j=0}^{m} f_{n, j} z^{j}=\sum_{k=0}^{\infty} \prod_{m, k} \widehat{P}_{k}(z),
$$

พThere

$$
\left|\prod_{m, k}\right|=\left|\sum_{j=0}^{\min (k, m)} f_{n, j} f_{k, j} \xi^{j}\right| \leq M\left(f_{n} ;|\xi| / r\right) \quad M\left(f_{k} ; r\right),
$$

and the number $r$ is chosen, in view of (2.7), such that

$$
\mathrm{T}^{\prime}<\mathrm{r}<\mathrm{n} \text {. }
$$

Now, if $\hat{\mathrm{F}}_{n}(\mathrm{C})$ is the Cannon sum of the set $\left\{\hat{\mathrm{P}}_{k}(z)\right\}$ for $\bar{D}(C)$, it follows from $(1.8),(5.2),(5.6),(5.7)$, and (5.8) that

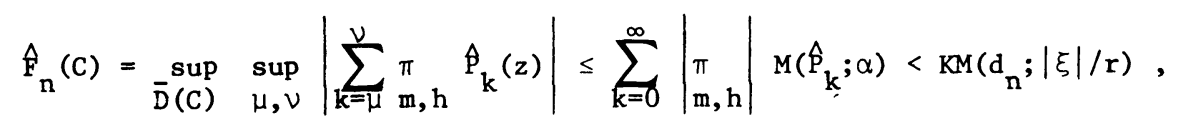

where $\mathrm{K}$ is a positive finite number independent of $\mathrm{n}$. Hence, for the Cannon function $\hat{K}(C)$, we should have, from (1.3), (1.8) and (5.8),

$$
\hat{K}(C)=\underset{k \rightarrow \infty}{\lim \sup }\left\{\hat{F}_{n}(C)\right\}^{1 / n} \leq E(|\xi| / r),
$$

and the required inequality (5.4) can be deduced from (5.9), and from the equation (2.9) by the choice of the number $r$.

The secind main result of the present work is formulated in the following theorem. THEOREM 5.2. For all forms of the Faber curve $C$, the transposed inverse set $\left\{\hat{P}_{k}(z)\right\}$ if (5.3) is not effective in $D(C)$. Moreover, there are certain Faber curves (C) for which the Cannon function $\hat{\kappa}(C)=\sigma$.

PROOF. The proof of the treorem is almost identical with that of Theorem 2 of [2[. In fact. if we write 


$$
w_{0}=\xi e i^{\omega} / n ; \sigma_{1}=\left|\psi\left(w_{0}\right)\right|,
$$

then, as in formula (6.8) of [2], we deduce from (2.6) that

$$
\sigma_{1}>\gamma
$$

Furthermore if $G(z)=1 /\left(w_{0}-z\right)$ and if the basic series associated with the Taylor expansion of $\mathrm{G}(\mathrm{z})$ is $\sum_{\mathrm{h}=0 \mathrm{~A}}^{\infty} \mathrm{P}_{\mathrm{k}}(\mathrm{z})$, then from (5.10), the analogue of (6.9) of [2] is

$$
A_{k}=\frac{1}{w_{0}} f_{k}\left(n e^{-i \omega}\right)
$$

Therefore, as in $[2 ;$ p. $217 ; 11.21-23]$, and by appeal to the relations $(1.6),(5.1)$, $(5.2),(5.5),(5.10),(5.11)$ and $(5.12)$, we can deduce that there exists a positive integer $k_{0}$ such that

$$
\left|A_{k}\right|\left|\hat{n}_{k}(\zeta)\right|>\frac{n L^{\prime \prime}}{2 \alpha \tau}
$$

for $k>k_{0}$, where $L^{\prime \prime}=\left|\psi^{\prime}(\xi / \zeta)\right|>0$. It follows that the set $\left\{\hat{P}_{k}(z)\right\}$ is not effective in $\bar{D}(C)$. Since, however, the Cannon function $\hat{K}(C)$ is bounded, by theorem 5.1 , then the set $\left\{\hat{\mathrm{P}}_{k}(z)\right\}$ cannot be effective in $D(C)$; as required.

By a discussion similar to that of $[2 ; \mathrm{p} .217 ; 11.29,30$ and p. $218 ; 11.1-5]$ we may conclude form (5.4) that $\hat{K}(C)=\sigma$ when $C$ is either a circle or a Cassini oval. The proof of theorem 5.2 is therefore complete.

\section{REFERENCES.}

1 NEWNS, W. F., On the Representation of Analytic Functions by Infinite Series, Phil. Trans. Roy. Soc. A 245. (1953) (429-468).

2 NASSIF, M., On the Transpose of Simple Sets of Polynomials Effective in Faber Regions, Pro. Amer. Math. Soc. 25. (1970) (209-219).

3 ADEPOJU, J. A., Basic Sets of Gonćarov Polynomials and Faber Regions, Ph. D. Thesis (1979) (Lagos).

4 WHITTAKER, J. M., Sur les Séries de Base de Polynomes Quelconques, (1949) (Paris).

5 NEWNS, W. F., The Range of Effectiveness of Basic Sets, Proc. London Math. Soc. 18. (1968) (745-767).

6 NEWNS, W. F., Product of two Simple Sets Effective in a Faber Region, Nederl. Akad. Wetensch. Proc. Ser A Indag. Math. 63. (1960) (187-191). 


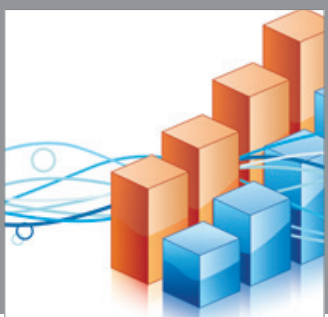

Advances in

Operations Research

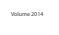

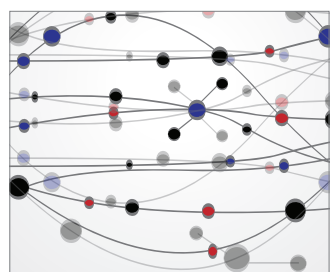

\section{The Scientific} World Journal
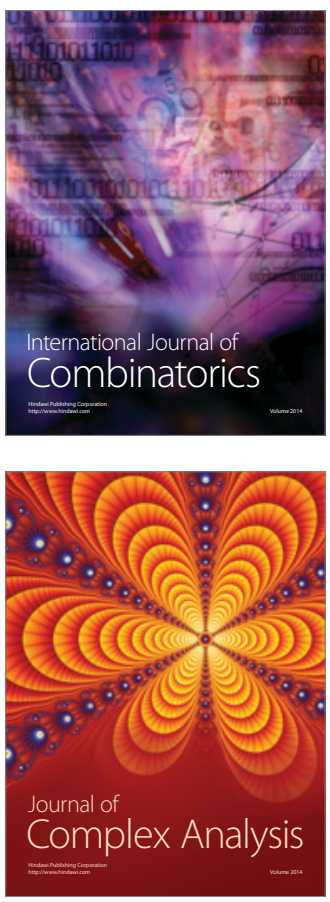

International Journal of

Mathematics and

Mathematical

Sciences
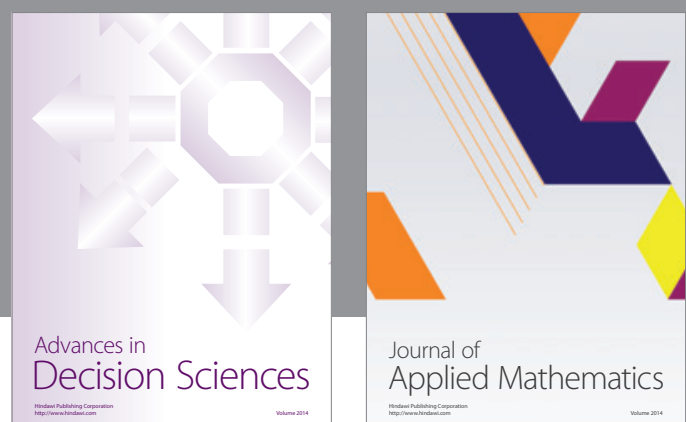

Journal of

Applied Mathematics
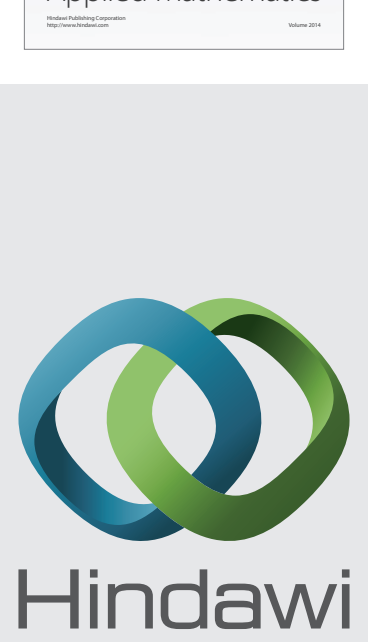

Submit your manuscripts at http://www.hindawi.com
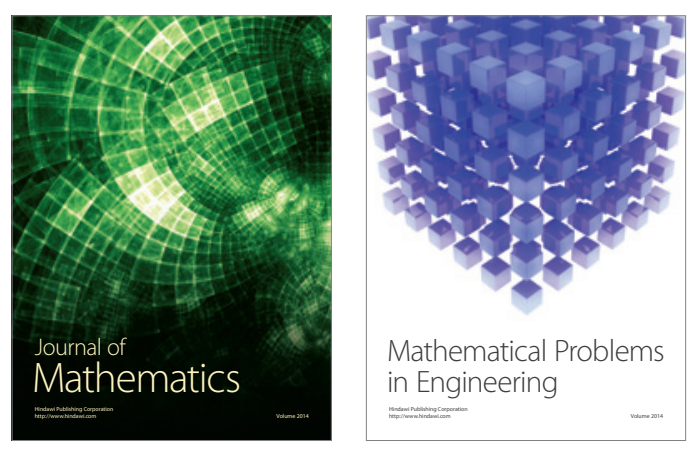

Mathematical Problems in Engineering
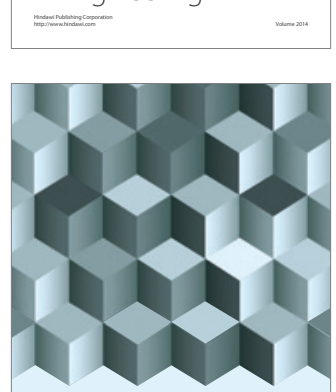

Journal of

Function Spaces
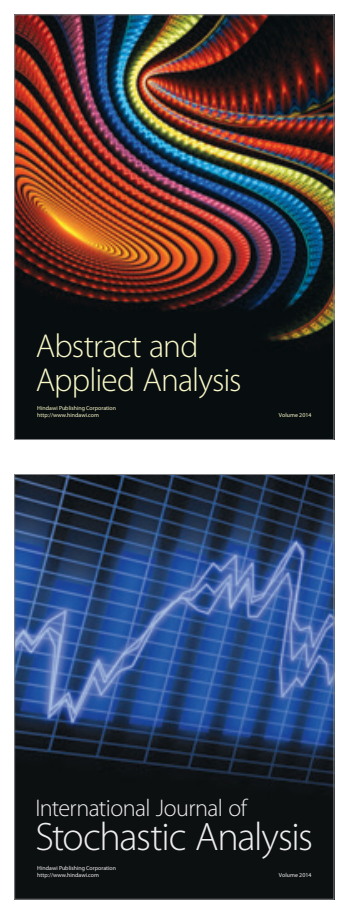

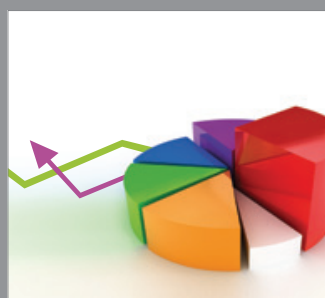

ournal of

Probability and Statistics

Promensencen
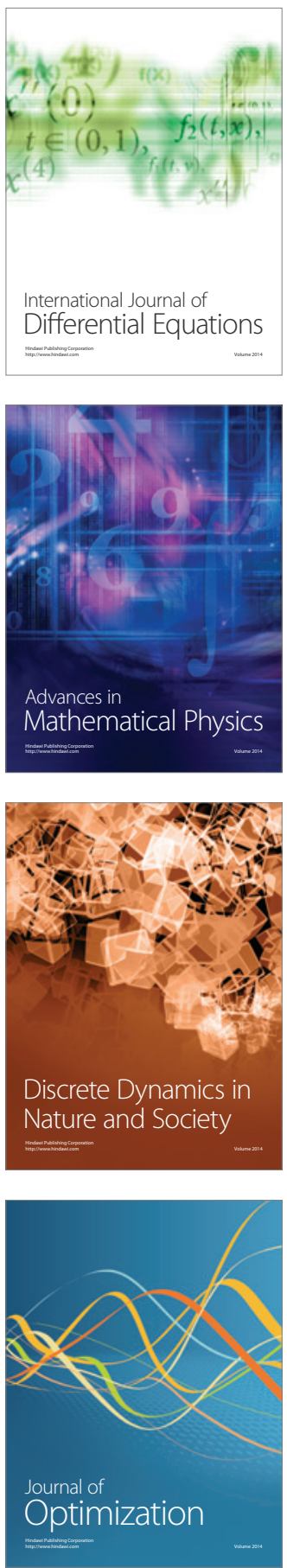